\title{
Quiste Renal Simple
}

\author{
Dra. Sonia Hannig X. ${ }^{1}$; Dra. Marisa del Valle M. ${ }^{1}$; Dra. Mónica Iglesias C. ${ }^{2}$ \\ Dra. Alejandra Cerón C. 3 ; Dr. César Izzo \$.4
}

\author{
Simple Renal Cysts
}

\begin{abstract}
Two patients with Simple Renal Cysts were studied and followed along a three years period. The first case was that of a 3 months old infant with a right abdominal mass, that had been discovered in the first month of life, and severe, persistent arterial hypertension that did not respond to treatment with propanolol, diazoxyde and hydrochlorothiazide: A solitary cyst without communications to the renal excretory apparatus was confirmed at surgery. The second patient had a left hemiabdominal mass that was detected at the Sth month of gestation, during a rutine echographic examination of his mother. In both cases a neprectomy was performed. Arterial hypertension subsided to normal two months after operation in case 1 and both patients were healthy at 42 months of age.

(Key words: Renal cysts. Abdominal tumors. Arterial hypertension. Prenatal diagnosis. Echography).
\end{abstract}

Los quistes renales simples son raros en los niffos, se han descrito alrededor de cincuenta casos desde 1834. Se observan con mayor frecuencia en adultos, habiéndose encontrado en el 2 a $4 \%$ de diversos estudios necrópsicos ${ }^{1,2}$.

Generalmente afectan al sexo masculino, ubicándose de preferencia en el riñón izquierdo, tanto en el polo superior como inferior; en la corteza o la médula renal, sin comunicarse con la pelvis renal. Son de tamaño variable, únicos o múltiples ${ }^{3,4}$.

La forma de presentación clínica más frecuente es la de una masa abdominal; ocasionalmente los pacientes consultan por dolor abdominal, hematurja, albuminuria $y$, raras veces por hipertensión arterial4,5.

En esta comunicación se descrjben dos pa. cientes estudiados en el Servicio de Pediatría del Hospital Sótero del Río y Hospital Calvo Mackenna, un recién nacido y un lactante menor, ambos portadores de quiste renal simple.

1. Becada. Departamento de Pediatría. Universidad Católica de Chile.

2. Cirujano Infantil. Servicio de Cirugía, Hospital Sótero del Río.

3. Médico Pediatra. Servicio de Pediatría, Hospital Sótero del Río.

4. Cirnjano Urólogo Infantil Jefe Servicio Cirugia Infantil Hospital Luis Calvo Mackenna.

\section{Casos Clínicos}

1. Varón de tres meses de edad que consultó por aumento de volumen abdominal notado al mes de vida, sin otros antecedentes mórbidos personales o familiares. En el hemiabdomen derecho tenía una masa de aproximadamente $15 \mathrm{~cm}$. de diámetro, lisa, elástica de borde redondo con leve peloteo. Presión arterial 190/130 mm Hg. Hemograma, velocidad de sedimentación de los glóbulos rojos, electrolitos plasmáticos y creatininemia normales. Urocultivo negativo. Microhematuria y moderada proteinuria. Exclusión del riffón derecho en la pielografía de eliminación. En la tomografía axial computarizada y la ecografía abdominal se encontró evidencia de una masa quistica en el riñón derecho. Vasoconstricción arteriolar severa en los fondos de ojos.

Fue tratado con propanolol ( $2 \mathrm{mg} \mathrm{x} \mathrm{kg} \mathrm{x} \mathrm{día),}$ Diazóxido ( $5 \mathrm{mg} \times \mathrm{kg} \times$ dosis) e Hidroclorotiazida $(5 \mathrm{mg} \times \mathrm{kg} \times$ dia) sin lograr reducir su hipertensión arterial, a pesar de lo cual no tuvo sintomas de encefalopatía. Por esta razón se realizó una pielostomia transparietal de urgencia, que dio salida a $600 \mathrm{ml}$ de líquido amarillento en 24 horas y pexmitió realizar inyección de medio de contraste que mostró, en el interior del quiste, una superficie irregular. A continuación se realizó una nefrectomía derecha, precedida por punción y drenaje del quiste, de cuyo contenido se aisló estaphilococcus aureus. 
El estudio anátomo patológico de la pieza extirpada no mostró alteraciones de la pelvis renal y el uréter. Existia un gran quiste, único, que nacía de la convexidad posterior del rifón; su superficie interna era irregular y por fuera tenía múltiples adherencias con el diafragma, la vena cava inferior y la glándula suprarrenal. Habia supuración en el interior del quiste, signos de hidronefrosis mínima y nefrjtis intersticial del rifión afectado.

El paciente sufrió una septicemia estafilocócica en el postoperatorjo, la que fue controlada con Cloxacilina y Gentamicina. La presión arterial se normalizó a los dos meses de edad, habiéndose mantenido el propanolol durante el primero y la hidrocloratiazida durante el segundo año de vida. Actualmente tiene 3 años y 6 meses, su crecimiento y su función renal son normales.

2. Recièn nacido de sexo masculino, pretérmino, adecuado para la edad gestacional. Durante el embarazo su madre tenía hipertensión arterial y diabetes gestacional. En una ecografía rutinaria a los $S$ meses de gestación se descubrió que tenía una masa abdominal que simulaba un riñón poliquístico. Nació por cesárea, pesó $2.930 \mathrm{~g}$ al nacer, en el período de recién nacido inmediato tuvo depresión respiratoria y taquipnea transitoria. Al ingresar se le palpaba una masa blanda que ocupaba todo el hemiabdomen izquierdo hasta la línea media. Hemograma, electrolitos plasmáticos, uremia, glicemia y sedimento urinario normales. En la pielografía de eliminación no se contrastó el riñón izquierdo.

A los 20 días de edad se realizó lumbotomia izquierda que permitió ver el riñón ipsilateral aplanado por un quiste de $5 \mathrm{~cm}$ de diámetro mayor, de superficie interna lisa, ubicado en su convexidad, respetando el sistema de eliminación. Pelvis y urêter eran normales. Se realizó nefrectomía izquierda. El estudio anatomopatológico confirmó que se trataba de un quiste renal simple. A los 3 anos $y$ medio estaba asintomático y su crecimiento era normal.

\section{DISCUSION}

Los quistes renales simples se han descrito en adultos pero rara vez alcanzan un tamaño sufjciente como para ser clínicamente evidentes durante la niñez ${ }^{4}$. Se han propuesto varias formas de clasificación para las lesiones quísticas renales; Braash y Henrich las dividen en quistes simples, de retención o inflamatorios, peripélvicos, secundarios a cambios patológicos en el tejido renal y poliquísticos. Los mismos autores subdividen los quistes simples en únicos, múltjples, multiloculares y hemorrágicos. Para ser ca. talogados como tales, deben ser uniloculares, comprometer sólo el parénqujma renal, su cavidad debe estar cubierta por epitelio, no debe contener elementos renales ni estar comunicada con la pelvis renal. Uréter y pelvis renal deben ser anatómicamente normales ${ }^{4}$.

La hipótesis más aceptada para explicar su patogenia es la de un trastorno del desarollo embrionario por ausencia de unión de la segunda generación de túbulos uriníferos y colectores, produciéndose así coleccione quísticas ${ }^{5}$.

Son más frecuentes en el riñón izquierdo y predominan en el sexo masculino. Ambos polos renales se comprometen con igual frecuencia. EI sintoma más común es la palpación de una masa abdominal, como en los casos presentados 5 .

Ia hipertensión arterial como hallazgo clínico es rara, y es posible que sea secundaria a alteraciones del parénquima, distorsión de los vasos renales producida por la masa, o ambas ${ }^{7}$ : en el primero de estos casos existia una gran elongación del pedículo vascular.

Los niños mayores pueden tener dolor abdominal; otros casos tienen también hematuria o proteinuria, como ocurrió en el primer paciente ${ }^{6}$, infecciones del tracto urinario, enuresis y retención urinaria ${ }^{12}$.

La infección secundaria del quiste se ha descrito excepcionalmente en adultos ${ }^{11}$. En el primer caso presentado, pese al hallazgo operatorio de supuración, no habían evidencias previas de infección.

La pielografía de eliminación permite en algunos casos identificar una masa renal, deformaciones en el aparato pielocaliciario o ambos, a yeces con exclusión renal del lado afectado como en nuestros pacientes. Otros métodos que ayudan en el diagnóstico son la ecografía y la tomografía axial computarizada, que permiten diferenciar entre masas sólidas y líquidas $\mathrm{s}^{9,10}$.

El diagnóstico diferencial de masas de origen renal en niños debe ser hecho entre hidronefrosis, tumor de Wilms, neuroblastoma, sinón multiquístico y duplicidad pielo-ureteral. El quiste renal simple tiene tan poca frecuencia que generalmente no se considera dentro de estas posibilidades. Además es difícil distinguirlo de otros tumores quísticos, incluso con los métodos descritos. En el primer caso presentado hubo dudas entre el tumor de Wilms y el quiste renal complicado. En el segundo se sospechó un riñón multicístico.

El tratamiento de elección es la resección del quiste; en los casos presentados había compro- 
miso del tejido renal adyacente, por lo que fue necesario efectuar una nefrectomía total, procedimiento recomendado en estas situaciones ${ }^{4.5}$.

Se ha sugerido un manejo más conservador que consiste en la punción del quiste renal bajo visión ecoscốpica ${ }^{5}$; sin embargo, comı en niffos pequeños los tumores son mucho más frecuentes, es recomendable hacer siempre laparotomia. $\mathrm{La}$ punción está indicada en nifros escolares con quistes renales más pequeños.

\section{RESUMEN}

Se presentan dos casos clínicos portadores de quiste renal simple con seguimiento clínico de tres años y medio cada uno.

El primer caso corresponde a un lactante de 3 meses de vida con un quiste renal simple supurado que evolucionó con hipertensión arterial severa que no respondía al tratamiento médico. El segundo, un recién nacido de 20 días cuyo diagnóstico se efectuó mediantẹ ecografía en el quinto mes de embarazo. El tratamiento se realizó en ambos pacientes extirpando el riñón afectado, obteniéndose normalización de la presión arterial, en el primero de ellos, dos meses después de la operación. Actualmente los niños están asintomáticos y su crecimiento y desarrollo son adecuados.

\section{REFERENCIAS}

1. Arev, J.B.: Cystic lesions of the kidney in infants and children. J. Pediatr. 54: 429, 1959.

2. Holl, W., Delporto, Gi; Keegan, G.i Tumier, R.: Case Report: Simple renal cyst in children J. Urol. 115: 165,1975 .

3. Garyin. D., Gehring, G,; Ball, $T .:$ Calcified solitary renal cyst in thilhood. J. Urol. 116: 644, 1976.

4. DeWeerd, $J$, Simon, $H$.: Simple renal cyst in children: Review of the literature and report of five cases. J. Urol. 75: 912, 1956.

5. Siegel, M., Mcalister, W.: Simple Cysts of kidney in children. J. Urol. 123: 75, 1980.

6. Noe, H., Lorimer, P.: Simple renal cyst manifest by proteinuria in children. J. Urol. 118: 854, 1977.

7. Babka, J., Cohen, M., Sode, J.: Solitary intrarenal cyst causing hypertension. N. Engl. J. Med. 291: $343,1974$.

8. Ravden, M. Zuckerman, H., Kay, C., Rosenfield. A.; Ablow, R., Rosenfield, N.: Evaluation of solitary simple renal cyst in children. 1 . Urol, 124: 904 , 1980.

9. Bearman, S., Sanders, R., Oh, K.: B-scan ultrasound in the evaluation of pediatric abdominal masses. Radiology 118: $111,1973$.

10. Sagel, S. Stanley, $R_{i}$ Levitt, $R_{+;}$Geisse, G.: Computed Tomography of the kidney. Radiology 124: $359,1977$.

11. Debruyne, F., Ypma, A., Moonen, W., Deloere, $\mathrm{K}$ : Infected Solitary cyst of the kidney. Review of the literature and three aditional cases. Acta Urol. Belg. 48: $21,1980$.

12. Gordon, R., Pollack, H., Popky, G., Duckott, J.: Simple Serous Cyst of the Kidney in Children. Radiology 131: 357, 1979 . 